\title{
RNGSSELIB: Program library for random number generation. More generators, parallel streams of random numbers and Fortran compatibility.
}

\author{
L.Yu. Barash, L.N. Shchur \\ Landau Institute for Theoretical Physics, 142432 Chernogolovka, Russia \\ Moscow Institute of Physics and Technology, 141700 Moscow, Russia \\ National Research University Higher School of Economics, Moscow Institute of \\ Electronics and Mathematics, 109028 Moscow, Russia
}

\begin{abstract}
In this update, we present the new version of the random number generator (RNG) library RNGSSELIB, which, in particular, contains fast SSE realizations of a number of modern and most reliable generators [1]. The new features are: i) Fortran compatibility and examples of using the library in Fortran; ii) new modern and reliable generators; iii) the abilities to jump ahead inside RNG sequence and to initialize up to $10^{19}$ independent random number streams with block splitting method.
\end{abstract}

\section{NEW VERSION PROGRAM SUMMARY}

Manuscript Title: RNGSSELIB: Program library for random number generation. More generators, parallel streams of random numbers and Fortran compatibility. Authors: L.Yu. Barash, L.N. Shchur

Program Title: RNGSSELIB

Journal Reference: Comput. Phys. Commun. 184 (2013) 2367

Catalogue identifier:

Licensing provisions:

Program summary URL: http://cpc.cs.qub.ac.uk/summaries/AEIT_v2_0.html Program obtainable from: CPC Program Library, Queens University, Belfast, N. Ireland

Licensing provisions: Standard CPC license, http://cpc.cs.qub.ac.uk/licence/licence.html

Email address: barash@itp.ac.ru (L.Yu. Barash) 
No. of lines in distributed program, including test data, etc.: 9299

No. of bytes in distributed program, including test data, etc.: 1768030

Distribution format: tar.gz

Programming language: C, Fortran

Computer: PC, laptop, workstation, or server with Intel or AMD processor

Operating system: Unix, Windows

RAM: 4 Mbytes

Number of processors used:

Supplementary material:

Keywords: Statistical methods, Monte Carlo, Random numbers, Pseudorandom numbers, Random number generation, Streaming SIMD Extensions

Classification: 4.13 Statistical Methods

External routines/libraries:

Subprograms used:

Catalogue identifier of previous version: AEIT_v1_0

Journal reference of previous version: Comput. Phys. Commun. 182 (2011) 1518

Does the new version supersede the previous version?: Yes

Nature of problem: Any calculation requiring uniform pseudorandom number generator, in particular, Monte Carlo calculations. Any calculation requiring parallel streams of uniform pseudorandom numbers.

Solution method: The library contains realization of the following modern and reliable generators: MT19937 [2], MRG32K3A 3], LFSR113 [4], GM19, GM31, GM61 [1], and GM29, GM55, GQ58.1, GQ58.3, GQ58.4 [5, 6]. The library contains both usual realizations and realizations based on SSE command set. Usage of SSE commands allows to substantially improve performance of all generators. Also, the updated library contains the abilities to jump ahead inside RNG sequence and to initialize independent random number streams with block splitting method for each of the RNGs.

Reasons for the new version: 1 . In order to implement Monte Carlo calculations, the implementation of independent streams of random numbers is necessary. Such implementation of initializing random number streams with block splitting method is added to the new version for each of the RNGs. Jumping ahead inside RNG sequence, which is necessary for the block splitting method, was also added for each of the RNGs. 2. Users asked us to add Fortran compatibility to the library. Fortran compatibility and the examples of using the library in Fortran for each of the RNGs are included in this version. 3. During last few years, the method of random number generation based on using the ensemble of transformations of two-dimensional torus, was essentially improved [5, 6]. Important properties, such 
as high-dimensional equidistribution, were established for the RNGs of this type. The proper choice of parameters was determined, which resulted in the validity of the high-dimensional equidistribution property, and, correspondingly, the new high-quality RNGs GM29, GM55.4, GQ58.1, GQ58.3, GQ58.4 were proposed. These RNGs are included now in the RNGSSELIB library.

Summary of revisions: 1 . We added Fortran compatibility and examples of using the library in Fortran for each of the generators. 2. New modern and reliable generators GM29, GM55.4, GQ58.1, GQ58.3, GQ58.4, which were introduced in [5] were added to the library. 3. The abilities to jump ahead inside RNG sequence and to initialize independent random number streams with block splitting method are added for each of the RNGs.

Restrictions: For SSE realizations of the generators, Intel or AMD CPU supporting SSE2 command set is required. In order to use the SSE realization for the Ifsr113 generator, CPU must support SSE4.1 command set.

Unusual features:

Additional comments: The function call interface has been slightly modified compared to the previous version in order to support Fortran compatibility. For each of the generators, RNGSSELIB supports the following functions, where rng should be replaced by the particular name of the RNG:

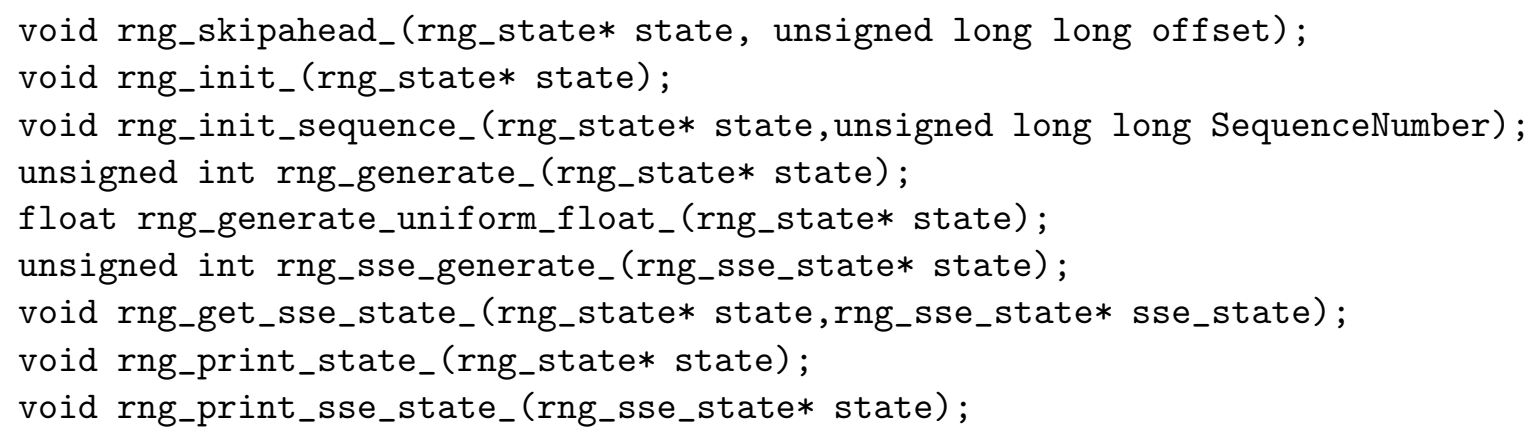

There are a few peculiarities for some of the RNGs. For example, the function void mt19937_skipahead_(mt19937_state* state, unsigned long longa, unsigned b); skips ahead $N=a \cdot 2^{b}$ numbers, where $N<2^{512}$, and the function void gm55_skipahead_(gm55_state* state, unsigned long long offset64, unsigned long long offset0); skips ahead $N=2^{64}$. offset $64+$ offset 0 numbers. The detailed function call 
Table 1: Initialization of pseudorandom streams for RNGs.

\begin{tabular}{|c|c|c|}
\hline Function initializing sequence & Number of sequences & Maximal length \\
\hline gm19_init_sequence_ & 1000 & $6 \cdot 10^{6}$ \\
\hline gm29_init_short_sequence_ & $10^{8}$ & $8 \cdot 10^{7}$ \\
\hline gm29_init_medium_sequence_ & $10^{6}$ & $8 \cdot 10^{9}$ \\
\hline gm29_init_long_sequence_ & $10^{4}$ & $8 \cdot 10^{11}$ \\
\hline gm31_init_short_sequence_ & $10^{9}$ & $8 \cdot 10^{7}$ \\
\hline gm31_init_medium_sequence_ & $10^{7}$ & $8 \cdot 10^{9}$ \\
\hline gm31_init_long_sequence_ & $10^{5}$ & $8 \cdot 10^{11}$ \\
\hline gm55_init_short_sequence_ & $10^{18}$ & $10^{10}$ \\
\hline gm55_init_long_sequence_ & $4 \cdot 10^{9}$ & $10^{20}$ \\
\hline gq58x1_init_short_sequence_ & $10^{8}$ & $8 \cdot 10^{7}$ \\
\hline gq58x1_init_medium_sequence_ & $10^{6}$ & $8 \cdot 10^{9}$ \\
\hline gq58x1_init_long_sequence_ & $10^{4}$ & $8 \cdot 10^{11}$ \\
\hline gq58x3_init_short_sequence_ & $2 \cdot 10^{8}$ & $8 \cdot 10^{7}$ \\
\hline gq58x3_init_medium_sequence_ & $2 \cdot 10^{6}$ & $8 \cdot 10^{9}$ \\
\hline gq58x3_init_long_sequence_ & $2 \cdot 10^{4}$ & $8 \cdot 10^{11}$ \\
\hline gq58x4_init_short_sequence_ & $3 \cdot 10^{8}$ & $8 \cdot 10^{7}$ \\
\hline gq58x4_init_medium_sequence_ & $3 \cdot 10^{6}$ & $8 \cdot 10^{9}$ \\
\hline gq58x4_init_long_sequence_ & $3 \cdot 10^{4}$ & $8 \cdot 10^{11}$ \\
\hline gm61_init_sequence_ & $1.8 \cdot 10^{19}$ & $10^{10}$ \\
\hline gm61_init_long_sequence_ & $4 \cdot 10^{9}$ & $3 \cdot 10^{25}$ \\
\hline lfsr113_init_sequence_ & $3.8 \cdot 10^{18}$ & $10^{10}$ \\
\hline lfsr113_init_long_sequence_ & $4 \cdot 10^{9}$ & $10^{24}$ \\
\hline mrg32k3a_init_sequence_ & $10^{19}$ & $10^{38}$ \\
\hline mt19937_init_sequence_ & $10^{19}$ & $10^{130}$ \\
\hline
\end{tabular}

interface can be found in the header files of the include directory. The examples of using the library can be found in the examples directory.

Table 1 shows maximal number of sequences and maximal length of each sequence for each function initializing pseudorandom stream. The algorithms used to jump ahead in the RNG sequence and to initialize parallel streams of pseudorandom numbers are described in detail in [7] and in [8].

This work was partially supported by Russian Foundation for Basic Research projects No. 12-07-13121 and 13-07-00570 and by the Supercomputing Center of Lomonosov Moscow State University [9]. 
Running time: Running time is of the order of $20 \mathrm{sec}$ for generating $10^{9}$ pseudorandom numbers with a PC based on Intel Core i7-940 CPU. Running time is analyzed in detail in [1] and in [5].

\section{References}

[1] L.Yu Barash, L.N. Shchur, RNGSSELIB: Program library for random number generation, SSE2 realization, Computer Physics Communications, 182 (7), 1518-1527 (2011); arXiv:1006.1235.

[2] M. Matsumoto and T. Tishimura, Mersenne Twister: A 623dimensionally equidistributed uniform pseudorandom number generator, ACM Trans. on Mod. and Comp. Simul. 8 (1), 3-30 (1998).

[3] P. L'Ecuyer, Good Parameter Sets for Combined Multiple Recursive Random Number Generators, Oper. Res. 47 (1), 159-164 (1999).

[4] P. L'Ecuyer, Tables of Maximally-Equidistributed Combined LFSR Generators, Math. of Comp., 68 (255), 261-269 (1999).

[5] L.Yu. Barash, Applying dissipative dynamical systems to pseudorandom number generation: Equidistribution property and statistical independence of bits at distances up to logarithm of mesh size, Europhysics Letters (EPL) 95, 10003 (2011).

[6] L.Yu. Barash, Geometric and statistical properties of pseudorandom number generators based on multiple recursive transformations // Springer Proceedings in Mathematics and Statistics, Springer-Verlag, Berlin, Heidelberg, Vol. 23, 265280 (2012).

[7] L.Yu. Barash, L.N. Shchur, On the generation of parallel streams of pseudorandom numbers, Programmnaya inzheneriya, 1 (2013) 24 (in Russian)

[8] L.Yu. Barash, L.N. Shchur, PRAND: GPU accelerated parallel random number generation library: Using most reliable algorithms and applying parallelism of modern GPUs and CPUs, submitted to Computer Physics Communications (2013) and to arXiv. 
[9] Voevodin Vl.V., Zhumatiy S.A., Sobolev S.I., Antonov A.S., Bryzgalov P.A., Nikitenko D.A., Stefanov K.S., Voevodin Vad.V., Practice of "Lomonosov" Supercomputer // Open Systems J. - Moscow: Open Systems Publ., 2012, no.7. (In Russian)

[10] Program codes can be downloaded from http://www. comphys.ru/barash/rngsselib_2.tar.gz 